\title{
Second-order ordinary differential systems with nonlocal Neumann conditions at resonance
}

\author{
Jean Mawhin ${ }^{1}$ - Katarzyna Szymańska-Dębowska²
}

Received: 4 June 2015 / Accepted: 2 September 2015 / Published online: 8 September 2015

(C) Fondazione Annali di Matematica Pura ed Applicata and Springer-Verlag Berlin Heidelberg 2015

Abstract Using topological degree arguments, several existence theorems are proved for the following system of nonlocal resonant boundary value problem

$$
x^{\prime \prime}=f\left(t, x, x^{\prime}\right), \quad x^{\prime}(0)=0, \quad x^{\prime}(1)=\int_{0}^{1} x^{\prime}(s) \mathrm{d} g(s)
$$

where $f:[0,1] \times \mathbb{R}^{k} \times \mathbb{R}^{k} \rightarrow \mathbb{R}^{k}$ is continuous and bounded, $g=\operatorname{diag}\left(g_{1}, \ldots, g_{k}\right) g_{j}$ : $[0,1] \rightarrow \mathbb{R}$ has bounded variation $(j=1, \ldots, k)$.

Keywords Nonlocal boundary value problem - Boundary value problem at resonance . Leray-Schauder fixed point theorem $\cdot$ Coincidence degree

Mathematics Subject Classification Primary 34B10 · Secondary 34B15 · 47H11

\section{Introduction}

Let $f:[0,1] \times \mathbb{R}^{k} \times \mathbb{R}^{k} \rightarrow \mathbb{R}^{k}$ be continuous and bounded, $g=\operatorname{diag}\left(g_{1}, \ldots, g_{k}\right)$ with $g_{j}:[0,1] \rightarrow \mathbb{R}$ having bounded variation, and let us consider the boundary value problem on $[0,1]$

Dedicated in the memory of Gaetano Villari.

Jean Mawhin

jean.mawhin@uclouvain.be

Katarzyna Szymańska-Dębowska

katarzyna.szymanska-debowska@p.lodz.pl

1 Institut de Recherche en Mathématique et Physique, Université Catholique de Louvain, chemin du Cyclotron, 2, 1348 Louvain-la-Neuve, Belgium

2 Institute of Mathematics, Lódź University of Technology, ul. Wólczańska 215, 90-924 Lódź, Poland 


$$
x^{\prime \prime}=f\left(t, x, x^{\prime}\right), \quad x^{\prime}(0)=0, \quad x^{\prime}(1)=\int_{0}^{1} x^{\prime}(s) \mathrm{d} g(s) .
$$

Such a problem is called resonant because, when $f \equiv 0$, the linear problem

$$
x^{\prime \prime}=0, \quad x^{\prime}(0)=0, \quad x^{\prime}(1)=\int_{0}^{1} x^{\prime}(s) \mathrm{d} g(s)
$$

has the family of nontrivial solutions $x(t)=c$ with $c \in \mathbb{R}^{k}$. It was recently considered by Szymańska-Dębowska [10] in the special case of

$$
x^{\prime \prime}=f(t, x), \quad x^{\prime}(0)=0, \quad x^{\prime}(1)=\int_{0}^{1} x^{\prime}(s) \mathrm{d} g(s)
$$

with $f=f(t, x)$ such that the limit

$$
h(t, \xi):=\lim _{r \rightarrow \infty} f(t, r \xi)
$$

exists uniformly in $\xi \in S^{k-1}$, with $S^{k-1}$ the unit sphere in $\mathbb{R}^{k}$. The result of [10], presented here as Theorem 4.1, was proved there by considering an approximate sequence of perturbed problems with invertible linear part, solving each of those using Schauder fixed point theorem, getting a uniform bound for the corresponding solutions and going to the limit to deduce a solution of (1.3).

The class of functions $f$ considered in [10] was introduced in 1971 by Nirenberg [7] for elliptic systems, generalizing the one introduced in 1970 by Landesman and Lazer [1] for semilinear elliptic equations with resonant linear part. More recently, Ortega and Sánchez [8] have used a similar class of functions in their study of periodic solutions of differential systems of the form

$$
x^{\prime \prime}+c x^{\prime}+g(x)=p(t)
$$

In this paper, we first show that the result of [10] for problem (1.3) follows in a straightforward way from the simplest form of Leray-Schauder continuation theorem [2] (sometimes referred as Schaefer's fixed point theorem [9]), to some fixed point operator in $C^{1}\left([0,1], \mathbb{R}^{k}\right)$ constructed in Sect. 2 (Lemma 2.1). This approach provides a generalization of the existence theorem of [10] to some $f$ which may depend upon $x^{\prime}$, need not to belong to the LandesmanLazer-Nirenberg class, but satisfy a condition of the type introduced in 1966 by Villari [11] for periodic solutions of resonant third-order differential equations.

The use of more sophisticated techniques from coincidence degree theory [3,5] provides other existence conditions for problem (1.1) in terms of the non-vanishing of the Brouwer degree of some mapping in $\mathbb{R}^{k}$ depending upon $f$ and $g$ (Theorem 6.1). From this result follow also existence conditions for problem (1.3) with Landesman-Lazer-Nirenberg nonlinearities, in terms of the non-vanishing of the Brouwer degree of some mapping in $\mathbb{R}^{k}$ depending upon $h$ defined in (1.4) and $g$ (Theorem 7.1).

When $g \equiv 0$, problems (1.1) and (1.3) reduce to classical Neumann problems, for which existence results in the line of Theorem 6.1 can be traced to [4]. 


\section{Equivalent fixed point problem}

In order to study problem (1.1) using Leray-Schauder-type arguments, we embed problem (1.1) into the homotopy

$$
\begin{aligned}
x^{\prime \prime} & =\lambda f\left(t, x, x^{\prime}\right), x^{\prime}(0)=0, \\
x^{\prime}(1) & =\int_{0}^{1} x^{\prime}(s) \mathrm{d} g(s)+(1-\lambda) x(0) \quad(\lambda \in[0,1]) .
\end{aligned}
$$

Problem (2.1) reduces for $\lambda=1$ to (1.1), and for $\lambda=0$, to the linear problem

$$
x^{\prime \prime}=0, \quad x^{\prime}(0)=0, \quad x^{\prime}(1)=\int_{0}^{1} x^{\prime}(s) \mathrm{d} g(s)+x(0) .
$$

The first two equations imply that $x(t) \equiv x(0)$, so that $x^{\prime}(t) \equiv 0$, and the third one implies $x(0)=0:$ For $\lambda=0$, problem (2.1) only has the trivial solution.

The following lemma shows that, for each $\lambda \in[0,1]$, system $(2.1)$ can be written as a fixed point problem in $C^{1}\left([0,1], \mathbb{R}^{k}\right)$, equipped with the norm

$$
\|x\|=\max _{t \in[0,1]}|x(t)|+\max _{t \in[0,1]}\left|x^{\prime}(t)\right| .
$$

Lemma 2.1 For each $\lambda \in[0,1]$, problem (2.1) is equivalent to the fixed point problem in $C^{1}\left([0,1], \mathbb{R}^{k}\right)$

$$
\begin{aligned}
x(t)= & \lambda\left[x(0)+\int_{0}^{1} f\left(s, x(s), x^{\prime}(s)\right) \mathrm{d} s-\int_{0}^{1} \int_{0}^{s} f\left(\tau, x(\tau), x^{\prime}(\tau)\right) \mathrm{d} \tau \mathrm{d} g(s)\right. \\
& \left.+\int_{0}^{t} \int_{0}^{s} f\left(\tau, x(\tau), x^{\prime}(\tau)\right) \mathrm{d} \tau \mathrm{d} s\right] \quad(t \in[0,1]) .
\end{aligned}
$$

Proof If $x \in C^{2}\left([0,1], \mathbb{R}^{k}\right)$ is a solution of (2.1) for some $\lambda \in[0,1]$, then

$$
\begin{aligned}
x^{\prime}(t) & =\lambda \int_{0}^{t} f\left(s, x(s), x^{\prime}(s)\right) \mathrm{d} s, \\
\lambda \int_{0}^{1} f\left(s, x(s), x^{\prime}(s)\right) \mathrm{d} s & =\lambda \int_{0}^{1} \int_{0}^{s} f\left(\tau, x(\tau), x^{\prime}(\tau)\right) \mathrm{d} \tau \mathrm{d} g(s)+(1-\lambda) x(0),
\end{aligned}
$$

so that

$$
\begin{aligned}
& x(t)=x(0)+\lambda \int_{0}^{t} \int_{0}^{s} f\left(\tau, x(\tau), x^{\prime}(\tau)\right) \mathrm{d} \tau \mathrm{d} s, \\
& x(0)=\lambda\left[x(0)+\int_{0}^{1} f\left(s, x(s), x^{\prime}(s)\right) \mathrm{d} s-\int_{0}^{1} \int_{0}^{s} f\left(\tau, x(\tau), x^{\prime}(\tau)\right) \mathrm{d} \tau \mathrm{d} g(s)\right],
\end{aligned}
$$

and hence $x(t)$ verifies (2.2). Conversely, if $x \in C^{1}\left([0,1], \mathbb{R}^{k}\right)$ is a solution of (2.2) for some $\lambda \in[0,1]$, then $x \in C^{2}\left([0,1], \mathbb{R}^{k}\right)$, and, from (2.2) we get, for all $t \in[0,1]$,

$$
x^{\prime}(t)=\lambda \int_{0}^{t} f\left(s, x(s), x^{\prime}(s)\right) \mathrm{d} s, x^{\prime \prime}(t)=\lambda f\left(t, x(t), x^{\prime}(t)\right) .
$$


Consequently, $x^{\prime}(0)=0$ and, taking $t=0$ in (2.2), we get

$$
\begin{aligned}
x(0)= & \lambda\left[x(0)+\int_{0}^{1} f\left(s, x(s), x^{\prime}(s)\right) \mathrm{d} s\right. \\
& \left.-\int_{0}^{1} \int_{0}^{s} f\left(\tau, x(\tau), x^{\prime}(\tau)\right) \mathrm{d} \tau \mathrm{d} g(s)\right],
\end{aligned}
$$

which, combined with the first equation in (2.3) taken in $t=1$, gives the second boundary condition in (2.1).

Now it is standard, using Ascoli-Arzelá's theorem, to show that the operator $\mathcal{F}$ : $C^{1}\left([0,1], \mathbb{R}^{k}\right) \rightarrow C^{1}\left([0,1], \mathbb{R}^{k}\right)$ defined by

$$
\begin{aligned}
\mathcal{F} x(t):= & x(0)+\int_{0}^{1} f\left(s, x(s), x^{\prime}(s)\right) \mathrm{d} s-\int_{0}^{1} \int_{0}^{s} f\left(\tau, x(\tau), x^{\prime}(\tau)\right) \mathrm{d} \tau \mathrm{d} g(s) \\
& +\int_{0}^{t} \int_{0}^{s} f\left(\tau, x(\tau), x^{\prime}(\tau)\right) \mathrm{d} \tau \mathrm{d} s \quad(t \in[0,1])
\end{aligned}
$$

is completely continuous, i.e., continuous and taking bounded subsets into relatively compact ones, and hence the same is true for the operator $(x, \lambda) \mapsto \lambda \mathcal{F} x$ from $C^{1}\left([0,1], \mathbb{R}^{k}\right) \times[0,1]$ to $C^{1}\left([0,1], \mathbb{R}^{k}\right)$.

\section{Conditions of Villari type}

In this section, we use the simplest version of Leray-Schauder's continuation theorem for $\mathcal{F}$ $[2,9]$.

Lemma 3.1 If there exists $R>0$ such that, for any $\lambda \in(0,1)$ and any possible fixed point $x$ of $\lambda \mathcal{F}$, one has $\|x\|<R$, then $\mathcal{F}$ has a fixed point $x$ such that $\|x\| \leq R$.

We first prove the following existence theorem, involving Villari-type conditions. $\langle\cdot, \cdot\rangle$ denotes the usual inner product in $\mathbb{R}^{k}$ and $|\cdot|$ the corresponding Euclidean norm.

Theorem 3.1 Assume that the following conditions hold:

(h1) There exists $M>0$ such that

$$
|f(t, x, y)| \leq M \text { for each }(t, x, y) \in[0,1] \times \mathbb{R}^{k} \times \mathbb{R}^{k} .
$$

(h2) There exists $r>0$ such that

$$
\left\langle x(0), \int_{0}^{1} f\left(s, x(s), x^{\prime}(s)\right) \mathrm{d} s-\int_{0}^{1} \int_{0}^{s} f\left(\tau, x(\tau), x^{\prime}(\tau)\right) \mathrm{d} \tau \mathrm{d} g(s)\right\rangle \leq 0
$$

for all $x \in C^{1}\left([0,1], \mathbb{R}^{k}\right)$ such that

$$
\min _{t \in[0,1]}|x(t)| \geq r \text { and } \max _{t \in[0,1]}\left|x^{\prime}(t)\right| \leq M .
$$

Then, problem (1.1) has at least one solution. 
Proof Let $\lambda \in(0,1)$ and let $x$ be a possible fixed point of $\lambda \mathcal{F}$. Then, $x$ satisfies the first equation in (2.3), and hence, using Assumption ( $h 1)$, we have, for all $t \in[0,1]$

$$
\left|x^{\prime}(t)\right| \leq \lambda\left|\int_{0}^{t} f\left(s, x(s), x^{\prime}(s)\right) \mathrm{d} s\right| \leq M .
$$

Consequently, if $\min _{t \in[0,1]}|x(t)| \geq r$, it follows from (2.4) and Assumption (h2) that,

$$
\begin{aligned}
0 & <(1-\lambda)|x(0)|^{2} \\
& =\lambda\left\langle x(0), \int_{0}^{1} f\left(s, x(s), x^{\prime}(s)\right) \mathrm{d} s-\int_{0}^{1} \int_{0}^{s} f\left(\tau, x(\tau), x^{\prime}(\tau)\right) \mathrm{d} \tau \mathrm{d} g(s)\right\rangle \\
& \leq 0
\end{aligned}
$$

a contradiction. Hence, there exists $\tau \in[0,1]$ such that $|x(\tau)|<r$, which, combined with (3.2), implies that, for all $t \in[0,1]$,

$$
|x(t)|=\left|x(\tau)+\int_{\tau}^{t} x^{\prime}(s) \mathrm{d} s\right| \leq|x(\tau)|+\int_{0}^{1}\left|x^{\prime}(s)\right| \mathrm{d} s<r+M,
$$

From (3.2) and (3.3), we get

$$
\|x\|<r+2 M:=R
$$

and the result follows from Lemma 3.1.

Remark 3.1 When $f\left(t, x, x^{\prime}\right)=f(t)$, it is easy to see that Assumption ( $\left.h 2\right)$ is equivalent to the necessary and sufficient condition

$$
\int_{0}^{1} f(s) \mathrm{d} s=\int_{0}^{1} \int_{0}^{s} f(\tau) \mathrm{d} \tau \mathrm{d} g(s)
$$

for the solvability of the non-homogeneous linear boundary value problem

$$
x^{\prime \prime}=f(t), \quad x^{\prime}(0)=0, \quad x^{\prime}(1)=\int_{0}^{1} x^{\prime}(s) \mathrm{d} g(s) .
$$

Remark 3.2 The conclusion of Theorem 3.1 still holds if, in Assumption (h2), condition (3.1) is replaced by

$$
\left\langle x(0), \int_{0}^{1} f\left(s, x(s), x^{\prime}(s)\right) \mathrm{d} s-\int_{0}^{1} \int_{0}^{s} f\left(\tau, x(\tau), x^{\prime}(\tau)\right) \mathrm{d} \tau \mathrm{d} g(s)\right\rangle \geq 0 .
$$

It suffices to replace homotopy (2.1) by the homotopy

$$
\begin{aligned}
x^{\prime \prime} & =\lambda f\left(t, x, x^{\prime}\right), x^{\prime}(0)=0, \\
x^{\prime}(1) & =\int_{0}^{1} x^{\prime}(s) \mathrm{d} g(s)-(1-\lambda) x(0) \quad(\lambda \in[0,1]) .
\end{aligned}
$$

For $\lambda=1$, we still get problem (1.1), and for $\lambda=0$, we obtain the problem

$$
x^{\prime \prime}=0, x^{\prime}(0)=0, x^{\prime}(1)=\int_{0}^{1} x^{\prime}(s) \mathrm{d} g(s)-x(0),
$$


which again has only the trivial solution. The corresponding fixed point operator is given by

$$
\begin{aligned}
\mathcal{G} x(t):= & x(0)-\int_{0}^{1} f\left(s, x(s), x^{\prime}(s)\right) \mathrm{d} s+\int_{0}^{1} \int_{0}^{s} f\left(\tau, x(\tau), x^{\prime}(\tau)\right) \mathrm{d} \tau \mathrm{d} g(s) \\
& +\int_{0}^{t} \int_{0}^{s} f\left(\tau, x(\tau), x^{\prime}(\tau)\right) \mathrm{d} \tau \mathrm{d} s \quad(t \in[0,1])
\end{aligned}
$$

and the proof of the variant of Theorem 3.1 proceeds like the one of this theorem, leading to the contradiction

$$
\begin{aligned}
0 & <(1-\lambda)|x(0)|^{2} \\
& =-\lambda\left\langle x(0), \int_{0}^{1} f\left(s, x(s), x^{\prime}(s)\right) \mathrm{d} s-\int_{0}^{1} \int_{0}^{s} f\left(\tau, x(\tau), x^{\prime}(\tau)\right) \mathrm{d} \tau \mathrm{d} g(s)\right\rangle \\
& \leq 0 .
\end{aligned}
$$

\section{Landesman-Lazer-Nirenberg nonlinearities}

The following lemma will be useful for proving existence theorems when $f(t, x)$ belongs to the Landesman-Lazer-Nirenberg class. Let $f:[0,1] \times \mathbb{R}^{k} \rightarrow \mathbb{R}^{k}$ be continuous.

Lemma 4.1 Assume that, the following conditions hold.

(H1) For each $t \in[0,1]$, the limit (1.4) exists uniformly in $\xi \in S^{k-1}$.

If there exists a sequence $\left(x_{n}\right)$ in $C^{1}\left([0,1], \mathbb{R}^{k}\right)$ such that $\inf _{t \in[0,1]}\left|x_{n}(t)\right| \geq n$ and such that the sequence $\left(y_{n}\right):=\left(\frac{x_{n}}{\left|x_{n}\right|}\right)$ converges uniformly on $[0,1]$ to $\eta \in S^{k-1}$, then, for each $t \in[0,1]$,

$$
\lim _{n \rightarrow \infty} f\left(t, x_{n}(t)\right)=h(t, \eta)
$$

Proof Let $\left(x_{n}\right)$ be the sequence in $C^{1}\left([0,1], \mathbb{R}^{k}\right)$ given in the assertion. Given $\varepsilon>0$, for each $t \in[0,1]$, one can find, by Assumptions (H1), a positive integer $N_{1}$ such that, for all $n \geq N_{1}$, and for all $\xi \in S^{k-1}$, one has

$$
\left|f\left(t,\left|x_{n}(t)\right| \xi\right)-h(t, \xi)\right| \leq \frac{\varepsilon}{2} .
$$

By the continuity of $h$, one can find a positive integer $N_{2}$ such that, for all $n \geq N_{2}$

$$
\left|h\left(t, y_{n}(t)\right)-h(t, \eta)\right| \leq \frac{\varepsilon}{2} .
$$

Consequently, for $n \geq N_{0}=\max \left\{N_{1}, N_{2}\right\}$, one has

$$
\begin{aligned}
\left|f\left(t, x_{n}(t)\right)-h(t, \eta)\right| & =\mid f\left(t,\left|x_{n}(t)\right| y_{n}(t)\right)-h\left(t, y_{n}(t)\right)+h\left(t, y_{n}(t)-h(t, \eta) \mid\right. \\
& \leq \varepsilon,
\end{aligned}
$$

which shows that, for each $t \in[0,1],\left(f\left(t, x_{n}(t)\right)\right)$ converges to $h(t, \eta)$.

The following result is the one proved in a different way in [10].

Theorem 4.1 Assume that condition (H1) holds and that 
(H2) For all $\xi \in S^{k-1}$,

$$
\left\langle\xi, \int_{0}^{1} h(s, \xi) \mathrm{d} s-\int_{0}^{1} \int_{0}^{s} h(\tau, \xi) \mathrm{d} \tau \mathrm{d} g(s)\right\rangle<0 .
$$

Then, problem (1.3) has at least one solution.

Proof Assumption (H1) implies the existence of some $M>0$ such that

$$
|f(t, x)| \leq M \text { for all }(t, x) \in[0,1] \times \mathbb{R}^{k} .
$$

By Theorem 3.1, it suffices to prove that condition (h2) is satisfied for (1.3). If it is not the case, there exists a sequence $\left(x_{n}\right)$ in $C^{1}\left([0,1], \mathbb{R}^{k}\right)$ such that

$$
\min _{t \in[0,1]}\left|x_{n}(t)\right| \geq n, \quad \max _{t \in[0,1]}\left|x_{n}^{\prime}(t)\right| \leq M,
$$

and, for all $n \in \mathbb{N}$,

$$
\left\langle\frac{x_{n}(0)}{\left|x_{n}(0)\right|}, \int_{0}^{1} f\left(s, x_{n}(s)\right) \mathrm{d} s-\int_{0}^{1} \int_{0}^{s} f\left(\tau, x_{n}(\tau)\right) \mathrm{d} \tau \mathrm{d} g(s)\right\rangle>0 .
$$

Set $y_{n}(t)=\frac{x_{n}(t)}{\left|x_{n}(t)\right|} \quad(n \in \mathbb{N})$, so that $\left|y_{n}(t)\right|=1 \quad(n \in \mathbb{N})$, and

$$
\left|y_{n}^{\prime}(t)\right|=\left|\frac{x_{n}^{\prime}(t)}{\left|x_{n}(t)\right|}-\frac{\left\langle x_{n}(t), x_{n}^{\prime}(t)\right\rangle}{\left|x_{n}(t)\right|^{3}} x_{n}(t)\right| \leq 2 \frac{M}{n} \leq 2 M .
$$

By (4.4) and Ascoli-Arzelà's theorem, going if necessary to a subsequence, there exists $y \in C\left([0,1], \mathbb{R}^{k}\right)$ such that $\left(y_{n}\right)$ converges uniformly on $[0,1]$ to $y$. Furthermore, $|y(t)|=1$ for all $t \in[0,1]$, and, by (4.4) again, $\left(y_{n}^{\prime}\right)$ converges to 0 uniformly on $[0,1]$. Consequently, $y \in C^{1}\left([0,1], \mathbb{R}^{k}\right), y^{\prime}=0$ on $[0,1]$ and $y$ is constant, namely $y(t)=\eta \in S^{k-1}(t \in$ $[0,1])$. By Lemma 4.1, for each $t \in[0,1],\left(f\left(t, x_{n}(t)\right)\right)$ converges to $h(t, \eta)$, and Lebesgue dominated convergence theorem implies that

$$
\begin{aligned}
\int_{0}^{1} f\left(t, x_{n}(t)\right) \mathrm{d} t & \rightarrow \int_{0}^{1} h(t, \eta) \mathrm{d} t \\
\int_{0}^{1} \int_{0}^{s} f\left(\tau, x_{n}(\tau)\right) \mathrm{d} \tau \mathrm{d} g(s) & \rightarrow \int_{0}^{1} \int_{0}^{s} h(\tau, \eta) \mathrm{d} \tau \mathrm{d} g(s),
\end{aligned}
$$

which introduced in (4.3) gives the contradiction

$$
0 \leq\left\langle\eta, \int_{0}^{1} h(t, \eta) \mathrm{d} t-\int_{0}^{1} \int_{0}^{s} h(\tau, \eta) \mathrm{d} \tau \mathrm{d} g(s)\right\rangle<0 .
$$

Remark 4.1 By using Remark 3.2, one can show that the conclusion is Theorem 4.1 remains valid if, in Assumption (H2), (4.2) is replaced by

$$
\left\langle\xi, \int_{0}^{1} h(s, \xi) \mathrm{d} s-\int_{0}^{1} \int_{0}^{s} h(\tau, \xi) \mathrm{d} \tau \mathrm{d} g(s)\right\rangle>0 .
$$




\section{Equivalent abstract semilinear equation}

In order to obtain more general existence conditions, we apply a continuation theorem from coincidence degree theory $[3,4]$. To this aim, we first write problem (1.1) in an equivalent form.

Lemma 5.1 $x$ is a solution of (1.1) if and only if $x \in C^{1}\left([0,1], \mathbb{R}^{k}\right)$ is a solution of the integro-differential boundary value problem

$$
x^{\prime}(t)=\int_{0}^{t} f\left(s, x(s), x^{\prime}(s)\right) \mathrm{d} s, \quad x^{\prime}(1)=\int_{0}^{1} \int_{0}^{s} f\left(\tau, x(\tau), x^{\prime}(\tau)\right) \mathrm{d} \tau \mathrm{d} g(s) .
$$

Proof If $x \in C^{2}\left([0,1], \mathbb{R}^{k}\right)$ is a solution of (1.1), then

$$
x^{\prime}(t)=\int_{0}^{t} f\left(s, x(s), x^{\prime}(s)\right) \mathrm{d} s, \quad x^{\prime}(1)=\int_{0}^{1} x^{\prime}(s) \mathrm{d} g(s),
$$

and hence (5.1) follows from inserting the first equation in the second one. Conversely, if $x \in C^{1}\left([0,1], \mathbb{R}^{k}\right)$ is a solution of $(5.1)$, then $x \in C^{2}\left([0,1], \mathbb{R}^{k}\right)$, and, by, respectively, differentiating the first equation, taking its value at 0 , and inserting the first equation in the third one, we get

$$
x^{\prime \prime}(t)=f\left(t, x(t), x^{\prime}(t)\right), \quad x^{\prime}(0)=0, \quad x^{\prime}(1)=\int_{0}^{1} x^{\prime}(s) \mathrm{d} g(s) .
$$

Let us define the linear operator $L: C^{1}\left([0,1], \mathbb{R}^{k}\right) \rightarrow C\left([0,1], \mathbb{R}^{k}\right) \times \mathbb{R}^{k}$ by

$$
D(L)=C^{1}\left([0,1], \mathbb{R}^{k}\right), L x=\left(x^{\prime}, x^{\prime}(1)\right) .
$$

$L$ is clearly continuous, and we denote its kernel by $N(L)$ and its range by $R(L)$. As

$$
L x=0 \quad \Leftrightarrow \quad x(t)=x(0) \quad(t \in[0,1]),
$$

$\operatorname{dim} N(L)=k$, and, for $(y, c) \in C\left([0,1], \mathbb{R}^{k}\right) \times \mathbb{R}^{k}$,

$$
L x=(y, c) \quad \Leftrightarrow \quad x^{\prime}(t)=y(t), x^{\prime}(1)=c
$$

has a solution if and only if $c=y(1)$, so that $R(L)=N(Q)$ with $Q$ the projector in $C\left([0,1], \mathbb{R}^{k}\right) \times \mathbb{R}^{k}$ defined by

$$
Q(y, c)=(0, c-y(1)) .
$$

Thus, $R(L)$ is closed and has codimension $k$, so that $L$ is a continuous Fredholm operator of index 0 . If we define $N: C^{1}\left([0,1], \mathbb{R}^{k}\right) \rightarrow C\left([0,1], \mathbb{R}^{k}\right) \times \mathbb{R}^{k}$ by

$$
N x(t)=\left(\int_{0}^{t} f\left(s, x(s), x^{\prime}(s)\right) \mathrm{d} s, \int_{0}^{1} \int_{0}^{s} f\left(\tau, x(\tau), x^{\prime}(\tau)\right) \mathrm{d} s \mathrm{~d} g(s)\right),
$$

it follows easily from standard arguments based upon Ascoli-Arzelá's theorem that $N$ is completely continuous. As $L$ has a continuous right inverse, $N$ is $L$-completely continuous in the sense of [5]. With those notations, problem (5.1), and hence problem (1.1), is equivalent to the abstract equation $L x=N x$. 


\section{More general Villari-type conditions and Brouwer degree}

We denote by $B(r)$ the open ball of center 0 and radius $r$ in $\mathbb{R}^{k}$, define the continuous mapping $\varphi: \mathbb{R}^{k} \rightarrow \mathbb{R}^{k}$ by

$$
\varphi(c)=\int_{0}^{1} \int_{0}^{s} f(\tau, c, 0) \mathrm{d} \tau \mathrm{d} g(s)-\int_{0}^{1} f(s, c, 0) \mathrm{d} s,
$$

and denote by $d_{B}[\varphi, B(r), 0]$, when it is defined, the Brouwer degree of $\varphi$ with respect to $B(r)$ and 0 [6]. We state the special case of Theorem IV.13 in [5] applied to $L-N$ with $L$ and $N$ defined above.

Lemma 6.1 Assume there exists $R>0$ such that the following conditions hold:

(c1) For each $\lambda \in(0,1)$, any possible solution of $L x=\lambda N x$ is such that $\|x\|<R$.

(c2) Any possible solution $c \in \mathbb{R}^{k}$ of $\varphi(c)=0$ is such that $|c|<R$.

(c3) $d_{B}[\varphi, B(R), 0] \neq 0$.

Then equation $L x=N x$ has at least one solution $x$ such that $\|x\| \leq R$.

We can now prove an existence theorem for (1.1) under a Villari-type condition.

Theorem 6.1 Assume that Assumption (h1) holds as well as the following conditions:

(a2) There exists $r>0$ such that

$$
\int_{0}^{1} \int_{0}^{s} f\left(\tau, x(\tau), x^{\prime}(\tau)\right) \mathrm{d} \tau \mathrm{d} g(s)-\int_{0}^{1} f\left(s, x(s), x^{\prime}(s)\right) \mathrm{d} s \neq 0
$$

for all $x \in C^{1}\left([0,1], \mathbb{R}^{k}\right)$ such that

$$
\min _{t \in[0,1]}|x(t)| \geq r \text { and } \max _{t \in[0,1]}\left|x^{\prime}(t)\right| \leq M .
$$

(a3) $d_{B}[\varphi, B(r), 0] \neq 0$.

Then, problem (1.1) has at least one solution.

Proof Let $\lambda \in(0,1)$ and $x \in C^{1}\left([0,1], \mathbb{R}^{k}\right)$ be a possible solution of $L x=\lambda N x$, i.e., of

$$
\begin{aligned}
& x^{\prime}(t)=\lambda \int_{0}^{t} f\left(s, x(s), x^{\prime}(s)\right) \mathrm{d} s, \\
& x^{\prime}(1)=\lambda \int_{0}^{1} \int_{0}^{s} f\left(\tau, x(\tau), x^{\prime}(\tau)\right) \mathrm{d} \tau \mathrm{d} g(s) .
\end{aligned}
$$

Then, by Assumption $(h 1)$, we have

$$
\left|x^{\prime}(t)\right| \leq M \quad(t \in[0,1]) .
$$

Now, it follows from (5.3) and (5.4) that

$$
Q N x=\int_{0}^{1} \int_{0}^{s} f\left(\tau, x(\tau), x^{\prime}(\tau)\right) \mathrm{d} s \mathrm{~d} g(s)-\int_{0}^{1} f\left(s, x(s), x^{\prime}(s)\right) \mathrm{d} s,
$$

and as $L x=\lambda N x(\lambda \in(0,1))$ implies $Q N x=0$, we have

$$
\int_{0}^{1} \int_{0}^{s} f\left(\tau, x(\tau), x^{\prime}(\tau)\right) \mathrm{d} s \mathrm{~d} g(s)-\int_{0}^{1} f\left(s, x(s), x^{\prime}(s)\right) \mathrm{d} s=0,
$$


Assumption (a2) implies the existence of $\tau \in[0,1]$ such that

$$
|x(\tau)|<r .
$$

Consequently, for all $t \in[0,1]$, it follows from (6.2) and (6.3) that

$$
|x(t)| \leq|x(\tau)|+\left|\int_{\tau}^{t} x^{\prime}(s) \mathrm{d} s\right|<r+M,
$$

and hence that

$$
\|x\|<r+2 M:=R .
$$

Thus, condition ( $(c 1)$ of Lemma 6.1 is satisfied, and the same is true, from Assumption (a2) restricted to constant functions, and from Assumption (a3), for conditions (c2) and (c3), because $d_{B}[\varphi, B(R), 0]=d_{B}[\varphi, B(r), 0] \neq 0$ follows from $\varphi(c) \neq 0$ for all $|c| \geq r$ and the excision property of Brouwer degree.

Remark 6.1 Theorem 3.1 with the slightly stronger Assumption $(h 2)$ with a strict inequality in (3.1) (or in its variant (3.4)) is a special case of Theorem 6.1. Indeed, (3.1) (respectively, (3.4)) then implies that Assumption ( $a 2$ ) of Theorem 6.1 holds and, furthermore, by taking constant functions $c$, that

$$
\langle c, \varphi(c)\rangle>0 \quad(\operatorname{resp} .\langle c, \varphi(c)\rangle<0)
$$

when $|c| \geq r$. This immediately implies, using the homotopy $(1-\lambda) c+\lambda \varphi(c)$ (resp. $-(1-\lambda) c+\lambda \varphi(c))$ and homotopy invariance of Brouwer degree, that

$$
\begin{aligned}
d_{B}[\varphi, B(r), 0]=d_{B}[I, B(r), 0] & =1 \\
\text { (resp. } \quad d_{B}[\varphi, B(r), 0]=d_{B}[-I, B(r), 0] & \left.=(-1)^{k}\right),
\end{aligned}
$$

so that Assumption (a3) of Theorem 6.1 is verified.

\section{Landesman-Lazer-Nirenberg nonlinearities and Brouwer degree}

When $f$ belongs to the Landesman-Lazer-Nirenberg class, we can deduce from Theorem 6.1 new conditions of existence involving Brouwer degree. Let $\psi: S^{k-1} \rightarrow \mathbb{R}^{k}$ be the continuous mapping defined by

$$
\psi(\xi)=\int_{0}^{1} \int_{0}^{s} h(\tau, \xi) \mathrm{d} \tau \mathrm{d} g(s)-\int_{0}^{1} h(s, \xi) \mathrm{d} s,
$$

where $h$ is defined in (1.4), and recall that when $0 \notin \psi\left(S^{k-1}\right)$, the Brouwer degree $d_{B}\left[\psi, S^{k-1}\right]$ is defined as the Brouwer degree $d_{B}[\Psi, B(1), 0]$ of any continuous extension $\Psi$ of $\psi$ to the closed unit ball $\bar{B}(1)$.

Theorem 7.1 Assume that Assumption (H1) holds as well as the following conditions:

(A2) $\psi(\xi) \neq 0$ for each $\xi \in S^{k-1}$.

(A3) $d_{B}\left[\psi, S^{k-1}\right] \neq 0$.

Then, problem (1.3) has at least one solution. 
Proof It follows from Assumption (H1) that Assumption $(h 1)$ of Theorem 3.1 applied to (1.3) holds for some $M>0$. If Assumption (a2) of Theorem 6.1 does not hold, there exists a sequence $\left(x_{n}\right)$ in $C^{1}\left([0,1], \mathbb{R}^{k}\right)$ such that

$$
\min _{t \in[0,1]}\left|x_{n}(t)\right| \geq n, \quad \max _{t \in[0,1]}\left|x_{n}^{\prime}(t)\right| \leq M,
$$

and, for all $n \in \mathbb{N}$,

$$
\int_{0}^{1} \int_{0}^{s} f\left(\tau, x_{n}(\tau)\right) \mathrm{d} \tau \mathrm{d} g(s)-\int_{0}^{1} f\left(s, x_{n}(s)\right) \mathrm{d} s=0 .
$$

Set $y_{n}(t)=\frac{x_{n}(t)}{\left|x_{n}(t)\right|} \quad(n \in \mathbb{N})$. Proceeding like in the proof of Theorem 4.1, we find that, going if necessary to a subsequence, $\left(y_{n}\right)$ converges uniformly on $[0,1]$ to some constant $\eta \in S^{k-1}$, and

$$
\begin{aligned}
\int_{0}^{1} f\left(t, x_{n}(t)\right) \mathrm{d} t & \rightarrow \int_{0}^{1} h(t, \eta) \mathrm{d} t \\
\int_{0}^{1} \int_{0}^{s} f\left(\tau, x_{n}(\tau)\right) \mathrm{d} \tau \mathrm{d} g(s) & \rightarrow \int_{0}^{1} \int_{0}^{s} h(\tau, \eta) \mathrm{d} \tau \mathrm{d} g(s),
\end{aligned}
$$

which introduced in (7.1) gives

$$
\int_{0}^{1} \int_{0}^{s} h(\tau, \eta) \mathrm{d} \tau \mathrm{d} g(s)-\int_{0}^{1} h(t, \eta) \mathrm{d} t=0,
$$

a contradiction to Assumption (A2). Now, it follows from Assumption (a2) that

$$
\varphi(c) \neq 0 \text { for }|c| \geq r
$$

and hence, by the excision property of Brouwer degree, for all $\rho \geq r$,

$$
d_{B}[\varphi, B(\rho), 0]=d_{B}[\varphi, B(r), 0] .
$$

Consequently, as $\varphi(\rho \cdot)$ on $\bar{B}(1)$ is a continuous extension of $\varphi(\rho \cdot)$ on $S^{k-1}$, we have, for $\rho \geq r$

$$
d_{B}\left[\varphi(\rho \cdot), S^{k-1}\right]=d_{B}[\varphi(\rho \cdot), B(1), 0]=d_{B}[\varphi, B(\rho), 0] .
$$

On the other hand, if follows from Assumption ( $H 1)$ and Lebesgue dominated convergence theorem and Assumption $(A 2)$ that

$$
\lim _{\rho \rightarrow \infty} \varphi(\rho \xi)=\psi(\xi) \neq 0 \quad \text { uniformly in } \xi \in S^{k-1},
$$

which implies that, by the invariance of Brouwer degree under small perturbations, there exists $R \geq r$ such that

$$
d_{B}\left[\varphi(R \cdot), S^{k-1}\right]=d_{B}\left[\psi, S^{k-1}\right] .
$$

It follows from (7.2), (7.3), (7.4), Assumption (A3) and the excision property of Brouwer degree, that

$$
d_{B}[\varphi, B(r), 0]=d_{B}[\varphi, B(R), 0]=d_{B}\left[\varphi(R \cdot), S^{k-1}\right]=d_{B}\left[\psi, S^{k-1}\right] \neq 0,
$$

and Assumption (a3) of Theorem 6.1 is satisfied. 
Remark 7.1 Theorem 4.1 follows from Theorem 7.1. Indeed, it follows from Assumption (H2) (respectively, from its alternative (4.5)), like in Remark 6.1, that $\psi(\xi) \neq 0$ for $\xi \in S^{k-1}$ and that

$$
\begin{aligned}
d_{B}\left[\psi, S^{k-1}\right] & =d_{B}\left[I, S^{k-1}\right]=1 \\
\text { (resp. } d_{B}\left[\psi, S^{k-1}\right] & \left.=d_{B}\left[-I, S^{k-1}\right]=(-1)^{k}\right) .
\end{aligned}
$$

The interest of the proof of Theorem 4.1 is that it depends only upon the simplest version of Leray-Schauder continuation theorem, which is equivalent to Schauder's fixed point theorem.

Example 7.1 For $n \geq 1$ an integer, $\alpha \in(0,1), p \in C\left([0,1], \mathbb{R}^{2}\right), a \in \mathbb{R}$, and using in $\mathbb{R}^{2}$ the complex notation, let us consider the boundary value problem

$$
z^{\prime \prime}=\frac{z^{n}}{1+|z|^{n}}-p(t), \quad z^{\prime}(0)=0, \quad z^{\prime}(1)=a z^{\prime}(\alpha) .
$$

This is a special case of (1.3) with

$$
k=2, \quad f(t, z)=\frac{z^{n}}{1+|z|^{n}}-p(t), \quad g_{1}(t)=g_{2}(t)=\left\{\begin{array}{l}
0 \text { if } t \in[0, \alpha] \\
a \text { if } t \in(\alpha, 1]
\end{array} .\right.
$$

For $\xi \in S^{1}, \quad t \in[0,1]$,

$$
h(t, \xi):=\lim _{r \rightarrow+\infty}\left[\frac{r^{n} \xi^{n}}{1+r^{n}}-p(t)\right]=\xi^{n}-p(t) \quad(t \in[0,1]) .
$$

Consequently, for $\xi \in S^{1}$,

$$
\begin{aligned}
\psi(\xi) & =\alpha a \xi^{n}-a \int_{0}^{\alpha} p(s) \mathrm{d} s-\xi^{n}+\int_{0}^{1} p(s) \mathrm{d} s \\
& =(\alpha a-1) \xi^{n}-\left(a \int_{0}^{\alpha} p(s) \mathrm{d} s-\int_{0}^{1} p(s) \mathrm{d} s\right) .
\end{aligned}
$$

Hence, if

$$
\alpha a \neq 1 \text { and } \frac{\left|a \int_{0}^{\alpha} p(s) \mathrm{d} s-\int_{0}^{1} p(s) \mathrm{d} s\right|}{|\alpha a-1|}<1
$$

we have

$$
d_{B}\left[\psi, S^{1}\right]=n \neq 0
$$

and hence problem (7.5) has at least one solution.

In the special case of Neumann problem, problem (7.5) with $a=0$, has at least one solution for all $p \in C\left([0,1], \mathbb{R}^{2}\right)$ such that

$$
\left|\int_{0}^{1} p(s) \mathrm{d} s\right|<1
$$

Notice that, in this case, if (7.5) has a solution $z$, then

$$
\left|\int_{0}^{1} p(s) \mathrm{d} s\right|=\left|\int_{0}^{1} \frac{z^{n}(s)}{1+|z(s)|^{n}} \mathrm{~d} s\right| \leq \int_{0}^{1} \frac{|z(s)|^{n}}{1+|z(s)|^{n}} \mathrm{~d} s<1,
$$

which shows that condition (7.6) is necessary and sufficient for the existence of a solution to the Neumann problem (7.5) with $a=0$. 
Remark 7.2 When $n \geq 2$, the existence result for problem (7.5) cannot be deduced from Theorem 4.1 because, as shown in Remark 7.1, the assumptions of Theorem 4.1 correspond to problem with associated Brouwer degree 1 or -1 .

\section{References}

1. Landesman, E.M., Lazer, A.C.: Nonlinear perturbations of linear elliptic boundary value at resonance. J. Math. Mech. 19, 609-623 (1970)

2. Leray, J., Schauder, J.: Topologie et équations fonctionnelles. Ann. Sci. École Norm. Supérieure (3) 51, 45-78 (1934)

3. Mawhin, J.: Equivalence theorems for nonlinear operator equations and coincidence degree theory for some mappings in locally convex topological vector spaces. J. Differ. Equ. 12, 610-636 (1972)

4. Mawhin, J.: Problèmes aux limites du type de Neumann pour certaines équations différentielles ou aux dérivées partielles non linéaires. In: Equations différentielles et fonctionnelles non linéaires, pp. 124-134. Hermann, Paris (1973)

5. Mawhin, J.: Topological Degree Methods in Nonlinear Boundary Value Problems, CBMS Regional Series in Mathematics No. 40. American Mathematical Society, Providence (1979)

6. Mawhin, J.: A simple approach to Brouwer degree based on differential forms. Adv. Nonlinear Stud. (4) 4, 535-548 (2004)

7. Nirenberg, L.: An application of generalized degree to a class of nonlinear problems. In: Troisième Colloque du C.B.R.M. d'analyse fonctionnelle, pp. 57-74. Vander, Louvain (1971)

8. Ortega, R., Sanchez, L.A.: Periodic solutions of forced oscillators with several degrees of freedom. Bull. Lond. Math. Soc. 34, 308-318 (2002)

9. Schaefer, H.H.: Ueber die Methode der a priori Schranken. Math. Ann. 129, 415-416 (1955)

10. Szymańska-Dębowska, K.: On a $k$-dimensional nonlocal boundary value problem at resonance. Electron. J. Differ. Equ. 2015, 1-8 (2015)

11. Villari, G.: Soluzioni periodiche di una classe di equazioni differenziali del terz'ordine quasi lineari. Ann. Mat. Pura Appl. (4) 73, 103-110 (1966) 